\title{
Investigation and Mathematical Modelling for Different Scattering Mechanisms in AIGaN/GaN HEMT
}

\author{
Md. Shafiqul Islam ${ }^{1}$, Sujjatul Islam ${ }^{1}$, Hasan Ahmed ${ }^{1}$, Abdul Ahad ${ }^{1}$ \\ ${ }^{1}$ Department of Electrical and Electronic Engineering, Bangladesh University of Engineering and Technology \\ Dhaka-1000, Bangladesh \\ islams@eee.buet.ac.bd; sujjatulislam@gmail.com; tonmoy1313@gmail.com; aahadeee11@gmail.com
}

\begin{abstract}
We investigated different scattering mechanisms in GaN High Electron Mobility Transistor with AlGaN barrier layer. This investigation leads to a quantum mechanical approach to develop mathematical model for different scattering limited mobilities. From these analytical results comparison between different scattering mechanisms were discussed under various two dimensional electron sheet charge densities. Finally total mobility curve was developed under both room (300K) and low (77K) temperatures for the first time.
\end{abstract}

Keywords: HEMT, AlGaN/GaN, 2DEG, Mobility, Scattering.

\section{Introduction}

AlGaN/GaN heterojunction can have extremely high 2DEG densities $\left(n_{s} \approx 2 \times 10^{13} \mathrm{~cm}^{-2}\right)$ with reasonably high room temperature mobility $\left(\mu \approx 1500 \mathrm{~cm}^{2} / \mathrm{V}\right.$.s) [1]. From the analysis of transport phenomenon it is seen that several scattering processes occur in 2DEG which limits the mobility of the transistor. Various scattering limited mobilities have been reported till date. These data indicate that total mobility depends upon the epitaxial layer growth technique, 2DEG density, temperature, spacer layer, barrier thickness, etc. Improvement of these factors resulted in higher mobilities. However, the carrier densities in the $\mathrm{AlGaN} / \mathrm{GaN} 2 \mathrm{DEGs}$ are typically an order of magnitude higher than that in $\mathrm{AlGaAs} / \mathrm{GaAs}$ 2DEGs, making nitride structures more suited for field-effect device applications [1]. The highest mobilities reported are for the lowest 2DEG densities. Thus the dominating scattering mechanisms at low temperatures should have a strong dependence on the 2DEG carrier density. Some paper indicate the turnaround of the trend for mobility at the lowest carrier concentrations [2]. Carrier densities lower than $n_{s}=10^{12} \mathrm{~cm}^{-2}$ are difficult to achieve in polar AlGaN/GaN heterojunctions due to the large polarization discontinuity at the hetero interface. The highest low-temperature mobilities are in the range of $\mu=7 \times 10^{4} \mathrm{~cm}^{2} / \mathrm{V}$.s at carrier densities in the range of $n_{s}=10^{12} \mathrm{~cm}^{-2}$. This is orders of magnitude lower than the highest mobilities reported for AlGaAs/GaAs modulation-doped 2DEGs, where the highest mobilities reported [3] are in the range $\mu \approx 10^{7} \mathrm{~cm}^{2} / \mathrm{V}$.s for carrier densities $n_{s} \approx$ $2 \times 10^{11} \mathrm{~cm}^{-2}$. The highest mobilities in AlGaAs/GaAs 2DEGs are remote ionized-impurity scattering limited. Thus the mobility increases with 2DEG density [3] which is opposite in trend to AlGaN/GaN 2DEGs.

\section{Scattering Mechanisms}

\subsection{Phonon Scattering}

Phonons are dominant scattering mechanisms for electrons at room temperature (>80K). Two important phonon scattering mechanisms are present in the AlGaN/GaN HEMT, the deformation coupling to longitudinal acoustic phonons and polar coupling to longitudinal optic phonon.

\subsubsection{Acoustic Phonon Scattering}

Though it is an inelastic scattering process the linear dispersion property makes acoustic phonon energy very low. Thus it can be assumed as elastic. The coupling of electron transport to acoustic phonons can be through deformation potential or piezoelectric components. However, it has been reported [4] that the piezo-electric component of acoustic phonon scattering in AlGaN/GaN 2DEGs is weaker than deformation potential scattering and can be neglected. Since the transverse modes are weaker than the longitudinal modes for deformation potential scattering, we can consider only the longitudinal mode acoustic scattering. The final acoustic phonon scattering rate is given by, 


$$
W_{a c}=\frac{\sqrt{2} m^{*^{3} / 2} a_{c}^{2} k_{B} T \sqrt{E_{0}}}{\pi \rho v_{s}^{2} \hbar^{4}}
$$

Here, $E_{0}=\left(\frac{9 \pi \hbar e^{2} n_{s}}{\left(8 \epsilon \sqrt{8 m^{*}}\right)}\right)^{2 / 3}$ where, $n_{s}$ is the 2DEG density, $m^{*}$ is the effective mass of electron, $T$ is the temperature, $v_{s}$ is the sound velocity and $a_{c}$, the $\Gamma$ valley conduction band deformation potential is assumed as $9.1 \mathrm{eV}$ [5]. The transport life time, $\tau_{a c}=\frac{1}{W_{a c}}$, the acoustic phonon limited mobility, $\mu_{a c}=\frac{e \tau_{a c}}{m^{*}}$.

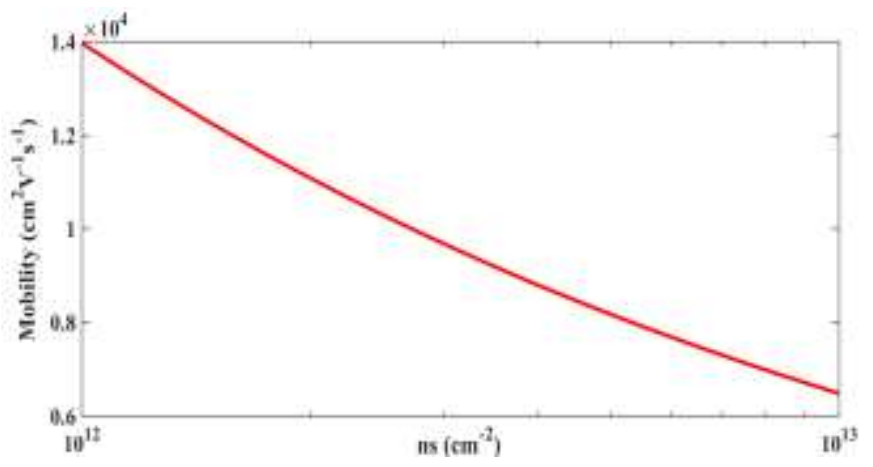

Fig. 1: Acoustic phonon limited mobility vs. 2DEG density curve.

Figure 1 shows that mobility decreases with the increase of sheet charge density. The sheet charge density increases with AlGaN barrier thickness, so mobility also decreases with barrier thickness.

\subsubsection{Optical Phonon Scattering}

In polar semiconductors the electron-longitudinal optical (LO) phonon scattering is the dominant inter subband scattering mechanism for separations of the sub-bands less than the LO phonon energy [6]. Polar optical phonon (POP) energy for the wurtzite GaN crystal is higher $\left(\hbar \omega_{\text {op }}=92 \mathrm{meV}\right)[1]$ than other III-V semiconductors. Scattering by polar optical phonons is highly inelastic so such a case demands the solution of Boltzmann equation by coupled equations for both emission and absorption thus making the relaxation-time approximation invalid [7]. Gelmont, Shur, and Stroscio [8] derived an analytic expression for the momentum relaxation rate in 2DEGs which is able to match experimental data over a wide temperature range rather accurately. The final scattering rate is given by,

$$
W(k)=\frac{V_{0}}{2 \pi m^{*} \hbar w_{0} v}\left(\frac{e e^{*}}{V_{0} \epsilon_{0}}\right)^{2}\left[n\left(w_{0}\right) \sinh ^{-1}\left(\frac{E_{k}}{\hbar w_{0}}\right)^{\frac{1}{2}}+\left(\mathrm{n}\left(w_{0}\right)+1\right) \sinh ^{-1}\left(\frac{E_{k}}{\hbar w_{0}}-1\right)^{\frac{1}{2}}\right]
$$

Where, $V_{0}$ is the scattering potential and $\epsilon^{*}=\epsilon_{0} \epsilon_{\mathrm{s}}$. Since the optical phonon energy is large $\left(\hbar \omega_{o p}>>k_{B} T\right)$, most carriers have energies lower than the optical phonon energy, thus blocking the emission of optical phonons. The absorption process dominates and is used to find the momentum relaxation time which is given by

$$
\frac{1}{\tau_{o p}}=\frac{e^{2} \omega_{0 p} m^{*} N_{B}(T) G\left(k_{0}\right)}{2 \epsilon^{*} q_{0} \hbar^{2} F(y)}
$$

Where, $q_{0}=\sqrt{\frac{2 m^{*} \hbar w_{0 p}}{\hbar^{2}}}$ is the polar optical phonon wave vector, $N_{B}$ is the Bose-Einstein distribution function and $F(y)=1+\frac{1-e^{-y}}{y}$ where, $y=\frac{\pi \hbar^{2} n_{2 D}}{m^{*} k_{B} T}$. 


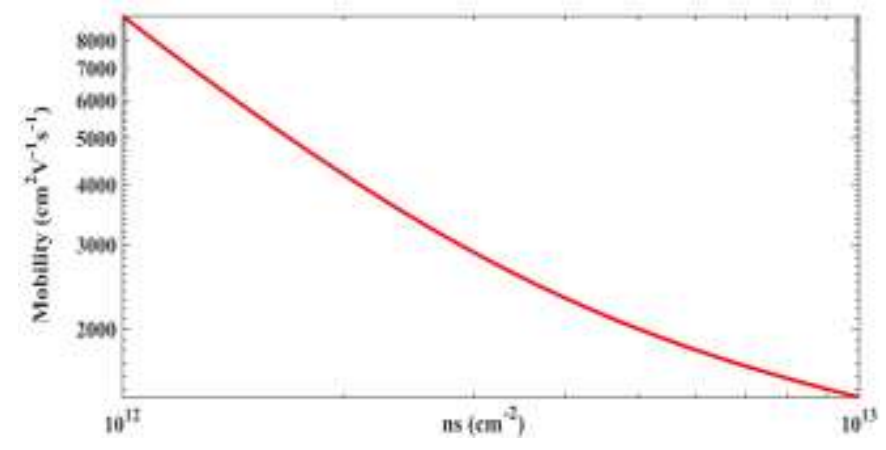

Fig. 2: Optical phonon limited mobility vs. 2DEG density curve.

Figure 2 shows the dependence of mobility on carrier density at $300 \mathrm{~K}$ due to optical phonon scattering which indicates a sharp decrease in mobility with the increase of $2 \mathrm{DEG}$ sheet charge density.

\subsection{Ionized Impurity Scattering}

\subsubsection{Remote lonized Impurity Scattering}

At the hetero-interface of the $\mathrm{AlGaN} / \mathrm{GaN}$ structure donor-like positively charged surface states are present [9]. The density of the surface states is equal to the sheet carrier charge density as a result of charge neutrality. This results in the formation of different Coulombic charge centers in different forms away from the heterointerface. These remote impurities affect the mobility of the charge carriers via Coulombic forces. This type of scattering is elastic and can be calculated via Fermi's Golden rule.

If the sheet charge density of remote donors is $n_{i m p}^{2 D}$, at a distance $d$ (barrier thickness of AlGaN), from the heterostructure interface, then the remote ionized impurity scattering limited mobility is given by,

$$
\mu_{r i o}=\frac{8 e\left(k_{F} d\right)^{3}}{\pi \hbar n_{i m p}^{2 D}}
$$

Where, $k_{F}=\sqrt{2 \pi n_{s}}$ which is the fermi wave vector.

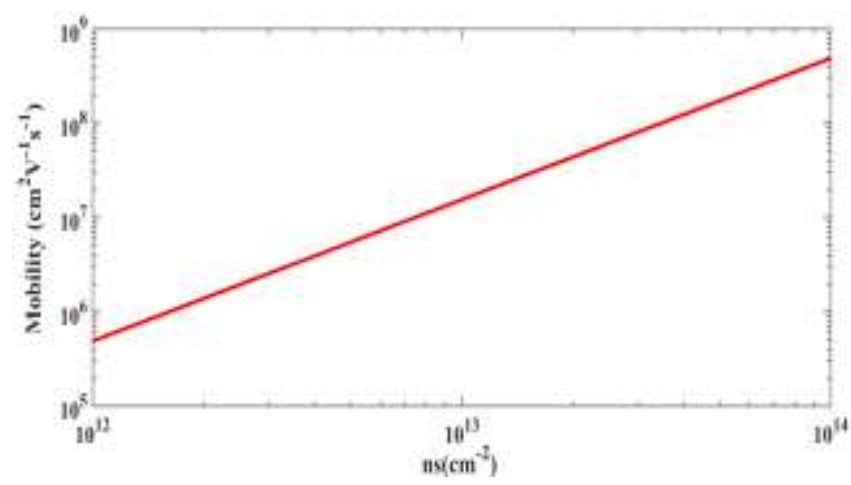

Fig. 3: Remote ionized impurity scattering limited mobility vs. 2DEG density.

Figure 3 demonstrates that the remote ionized impurity scattering limited mobility gradually increases with the increase in carrier concentration at $300 \mathrm{~K}$. 


\subsubsection{Background Residual Impurity Scattering}

In the $\mathrm{AlGaN} / \mathrm{GaN}$ structures unintentional residual background donors of order $10^{16} \mathrm{~cm}^{-3}$ are present [10]. These donors emerge during the growth process consisting of impurity atoms or vacant areas. The effect of this kind of scattering is comparatively less significant in $\mathrm{AlGaN} / \mathrm{GaN}$. The scattering rate for the presence of background residual donors of density $n_{i m p}^{3 D}$ is given by,

$$
\frac{1}{\tau_{\text {bio }}}=n_{\text {imp }}^{3 D} \frac{m^{*}}{2 \pi \hbar^{3} k_{F}^{3}}\left(\frac{e^{2}}{2 \epsilon_{0} \epsilon_{s}}\right)
$$

Where, $k_{F}=\sqrt{2 \pi n_{s}}$ which is the fermi wave vector. Figure 4 shows a linear increase in background residual impurity scattering limited mobility with 2DEG sheet charge density.

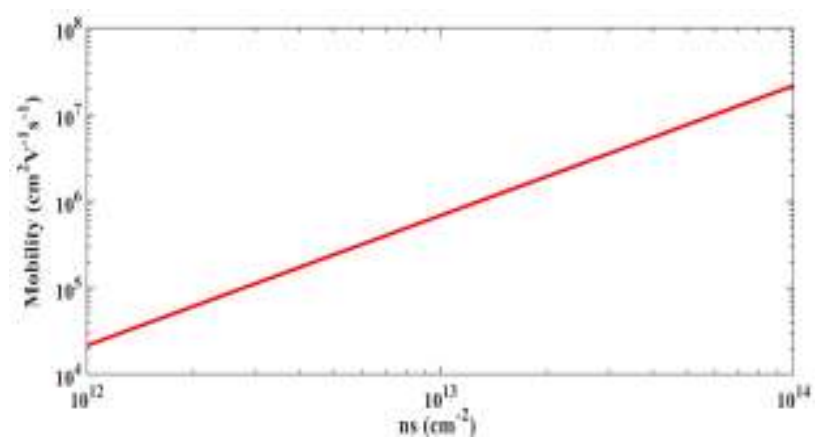

Fig. 4: Background residual impurity scattering limited mobility vs. 2DEG density.

\subsection{Interface Roughness Scattering}

Depending upon the fabrication techniques, the interface between two materials contains varying degree of roughness. The interface roughness causes potential bumps in the path of the carriers, causing the carriers to scatter. Scattering at rough interfaces can be severe if the 2DEG density is high, since the 2DEG tends to shift closer to the interface as the density increases.

At the heterointerface between $\mathrm{AlGaN}$ and $\mathrm{GaN}$, the electrons reside in a triangular quantum well. A number of models have been proposed to account for the form of interface roughness potential.

The interface roughness scattering rate is deduced as,

$$
\tau_{\text {int }}=\frac{m^{*} \Delta^{2} L^{2} e^{4}}{2 h^{3} \in_{s}^{2}}\left[I_{0}\left(\frac{k^{2} L^{2}}{2}\right)-I_{1}\left(\frac{k^{2} L^{2}}{2}\right)\right] \exp \left(\frac{-k^{2} L^{2}}{2}\right)\left(N_{\text {depl }}+\frac{n_{s}}{2}\right)^{2}
$$

Where, $I_{0}$ and $I_{1}$ are the modified Bessel functions, $\Delta$ represents the height of the interface bumps, $L$ represents their correlation length along the interface and $n_{s}$ is the 2DEG density. 


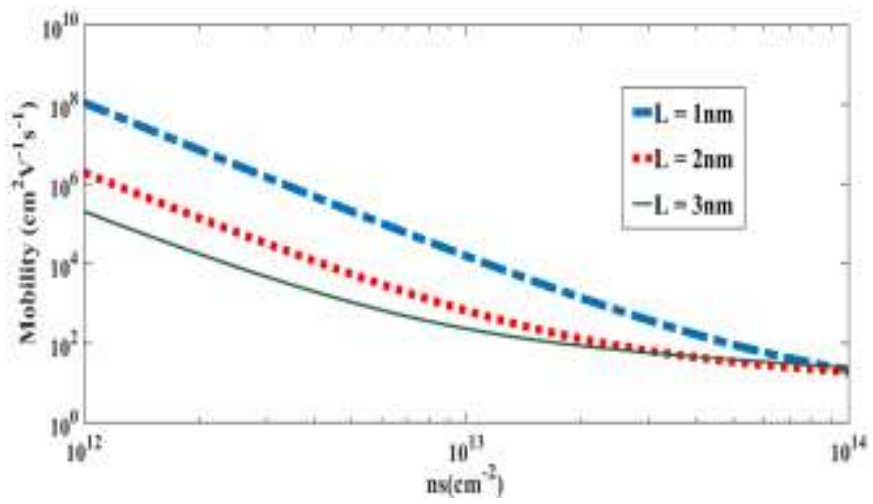

Fig. 5: Interface roughness scattering limited mobility vs. 2DEG density for $\Delta=2.5 \AA$.

A plot of interface roughness scattering limited mobility is shown in Fig. 5. Where, the average height of the bumps in the interface is assumed $\Delta=2.5 \AA$. The figure shows that the mobility decreases with the increase of 2DEG density because as more and more carriers concentrate at the hetero-interface, the interface roughness as forms of potential barriers are encountered more and as a result the carrier scattering rate increases. Also, increase of the correlation length decreases the mobility.

\subsection{Dislocation Scattering}

A dislocation is an irregularity within a crystal structure. Here, it refers to a defect on the atomic scale. The dislocation density is a measure of the number of dislocations in a unit volume of a crystalline material. These dislocations represent the large lattice mismatch with the selected substrate in the HEMT. In AlGaN/GaN system, the 2DEG contains a line dislocation density of order $10^{8} \mathrm{~cm}^{-2}$ passing through it. Due to these dislocations two scattering mechanisms are present, one due to charged dislocations and other is due to strain fields created by dislocations.

\subsubsection{Charge Dislocation Scattering}

The dislocations present in the GaN are electrically charged [11] and they introduce localized current paths [12]. It is also experimentally shown that in GaN these dislocations produce electronic states in the band gap [13][14]. These charged dislocations greatly affect the carrier mobility.

If there are $N_{\text {dis }}$ line dislocations piercing the $2 \mathrm{DEG}$ per unit area, the momentum relaxation time for the $2 \mathrm{DEG}$ is given by,

$$
\tau_{d i s}=\frac{\hbar^{3} \epsilon_{0}{ }^{2} \epsilon_{s}{ }^{2} c_{0}{ }^{2}}{N_{d i s} m^{*} e^{4} f^{2}} \frac{16 \pi k_{F}{ }^{4}}{\left(\frac{1.84 k_{F}}{q_{T F}}-0.25\right)}
$$

Where, the Fermi wave vector $k_{F}=\sqrt{2 \pi n_{s}}, q_{T F}=\frac{2}{a_{B}}$ where $a_{B}$ is the effective Bohr Radius, $n_{s}$ is the 2DEG density, $c_{0}$ is the lattice spacing in the [0001] direction of wurtzite GaN, $N_{\text {dis }}$ is the density of states and $f$ is the fraction of filled states. 


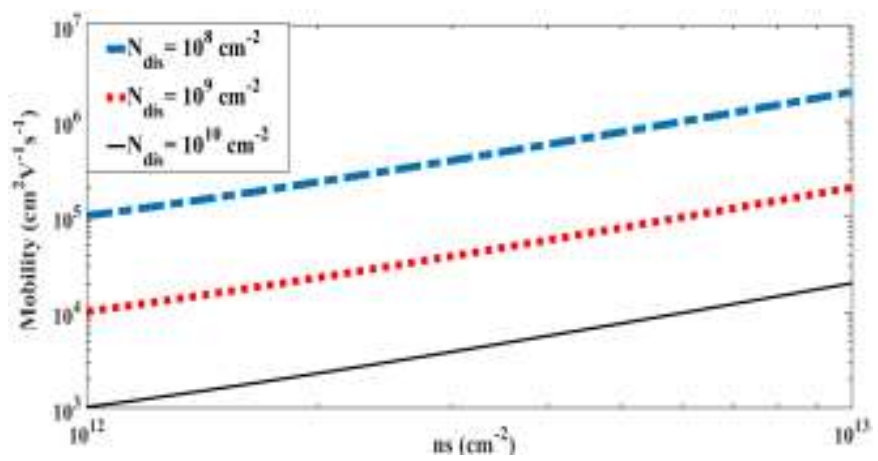

Fig. 6: Charge dislocation scattering limited mobility vs. 2DEG density at different dislocation densities.

Figure 6 shows that the charge dislocation scattering limited mobility increases with the increase of 2DEG density and decrease of the dislocation density causes in higher mobility.

\subsubsection{Strain Dislocation Scattering}

The dislocations in a semiconductor create a strain field around them which causes the crystal to lose its stability. Whether charged or uncharged, the effects of strain fields associated with the defects or dislocations in the semiconductor cause the scattering of the charge carriers [15].

Dislocation strain field scattering limited electron mobility given by,

$$
\tau_{d i s}=\frac{2 \pi k_{F}{ }^{2} \hbar^{3}}{N_{d i s} m^{* 2} b_{e}{ }^{2} a_{c}{ }^{2}}\left(\frac{1-\gamma}{1-2 \gamma}\right)^{2} \frac{1}{I\left(n_{s}\right)}
$$

Where, $b_{e}=a_{0}=3.189 \AA$ is the Burger's vector, $a_{c}$ is the conduction band deformation potential, $\gamma=0.3$ is the crystal Poisson's ratio [16], $k_{F}=\sqrt{2 \pi n_{s}}$ and the dimensionless integral $I\left(n_{s}\right)$ is given by,

$$
I\left(n_{s}\right)=\int_{0}^{1} \frac{d u}{\left(u+\frac{q_{T F}}{2 k_{F}}\right)^{2} \sqrt{1-u^{2}}}
$$

That is $I\left(n_{s}\right)$ is solely dependent on $n_{s}$. We require only the conduction band deformation potential for our calculation. The conduction band deformation potential from transport analysis to be $a_{C}=(9.1 \pm 0.7) \mathrm{eV}$ [16]. Their measured value is used for calculations here when, $N_{\text {dis }}=10^{9} \mathrm{~cm}^{-2}$.

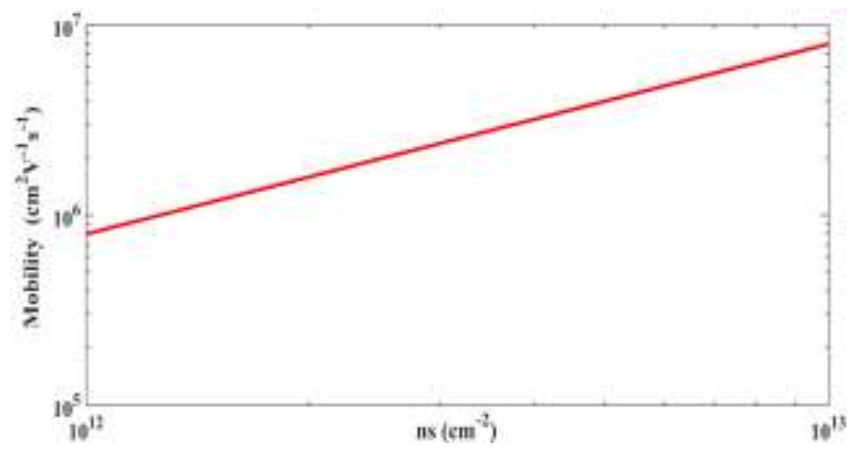

Fig. 7: Strain dislocation scattering limited mobility vs. 2DEG. 
Strain dislocation scattering limited mobility increases as the 2DEG increases (Fig. 7) which confirms that the effect of the dislocation caused strain gradually diminishes.

\subsection{Alloy Scattering}

The randomly varying alloy potential in the barrier results in the finite penetration of the 2DEG wave function into the barrier. With the modified Fang-Howard algebra [17], the momentum scattering rate is calculated as,

$$
\frac{1}{\tau_{\text {alloy }}}=\frac{3 \pi^{3}}{8 \hbar} \Omega \Delta U^{2} x(1-x) \frac{m^{*^{3 / 2}}\left(k_{B} T\right)^{1 / 2}}{0.75 \sqrt{2} \pi^{2} \hbar^{3}}
$$

Where $\Omega$ is the volume of the unit cell, $\Delta U$ is the scattering potential and $x$ is the mole fraction of AlGaN.

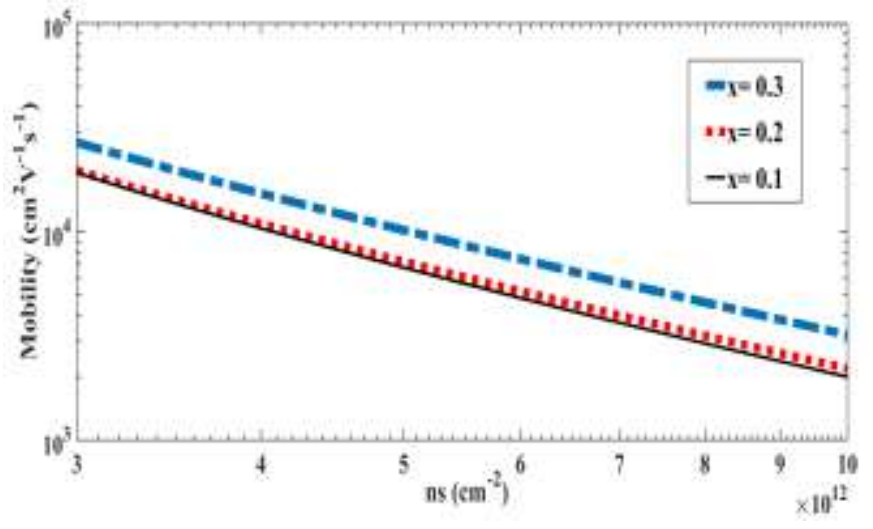

Fig. 8: Alloy scattering limited mobility vs. 2DEG density curve with different alloy compositions.

Figure 8 shows that as carrier density increases, the wave function gets pushed closer to the junction, leading to more penetration into the alloy barrier and hence stronger scattering, so mobility decreases. Low alloy concentrations of $\mathrm{Al}_{\mathrm{x}} \mathrm{Ga}_{1-\mathrm{x}} \mathrm{N}$ means reduced conduction-band discontinuity and large penetration of the 2DEG wave function into the barrier and so mobility decreases.

\section{Total Mobility}

We apply Matthiessen's rule for determining overall mobility curve. This rule dictates that if $\mu_{\text {tot }}$ is the total mobility, then $\frac{1}{\mu_{\text {tot }}}=\sum_{i=1}^{N} \frac{1}{\mu_{\mathrm{i}}}$

Where, $\mathrm{N}$ is the total number of scattering mechanisms. We get,

$$
\frac{1}{\mu_{\text {tot }}}=\frac{1}{\mu_{\text {ac }}}+\frac{1}{\mu_{\text {op }}}+\frac{1}{\mu_{\text {rio }}}+\frac{1}{\mu_{\text {bio }}}+\frac{1}{\mu_{\text {ir }}}+\frac{1}{\mu_{\text {alloy }}}+\frac{1}{\mu_{\text {cdis }}}+\frac{1}{\mu_{\text {sdis }}}
$$

This relation allows us to obtain the total mobility curve shown in Fig. 10 and Fig. 12. The figures show the variation of mobility with 2DEG sheet charge density calculated for the room temperature of $300 \mathrm{~K}$ and at low temperature of $77 \mathrm{~K}$. Figure 9 and Fig. 11 give a comparative view of total mobility at two temperatures. There is a peak value of mobility at a certain 2DEG density. Again increase in temperature decreases the mobility which is justified from the figures and it shows that temperature plays a crucial part in scattering. 


\subsection{Mobility at $300 \mathrm{~K}$}

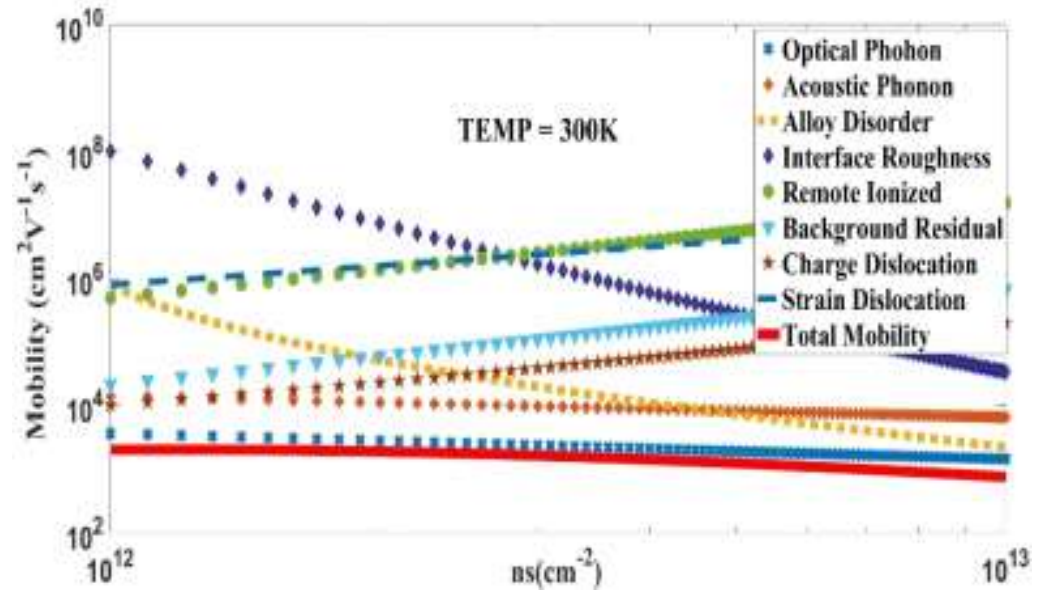

Fig. 9: Different mobilities plotted together against $2 \mathrm{DEG}$ density at $300 \mathrm{~K}$.

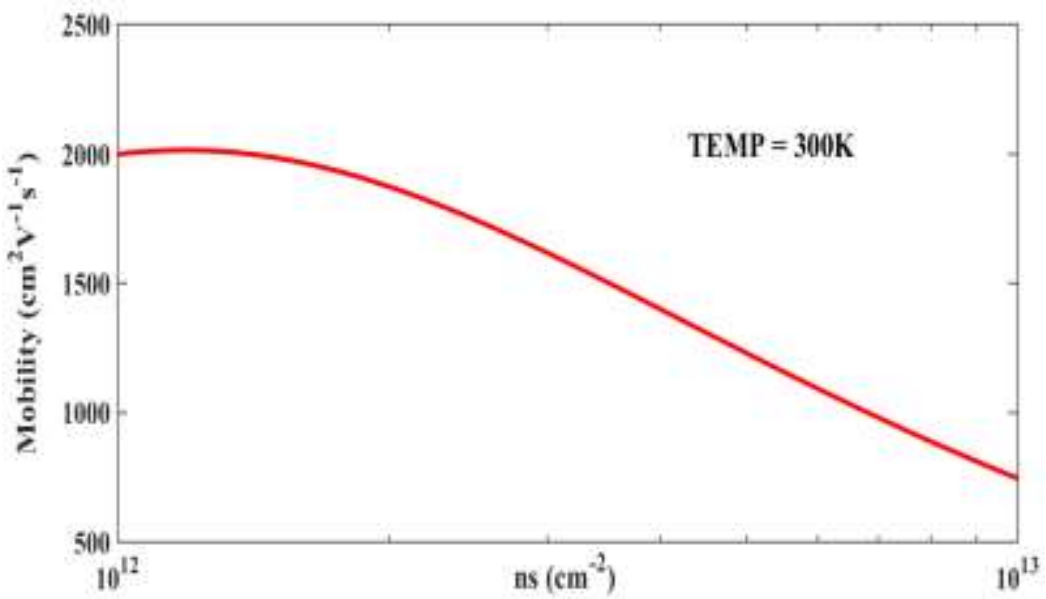

Fig. 10: Total mobility vs. 2 DEG density at $300 \mathrm{~K}$. 


\subsection{Mobility at $77 \mathrm{~K}$}

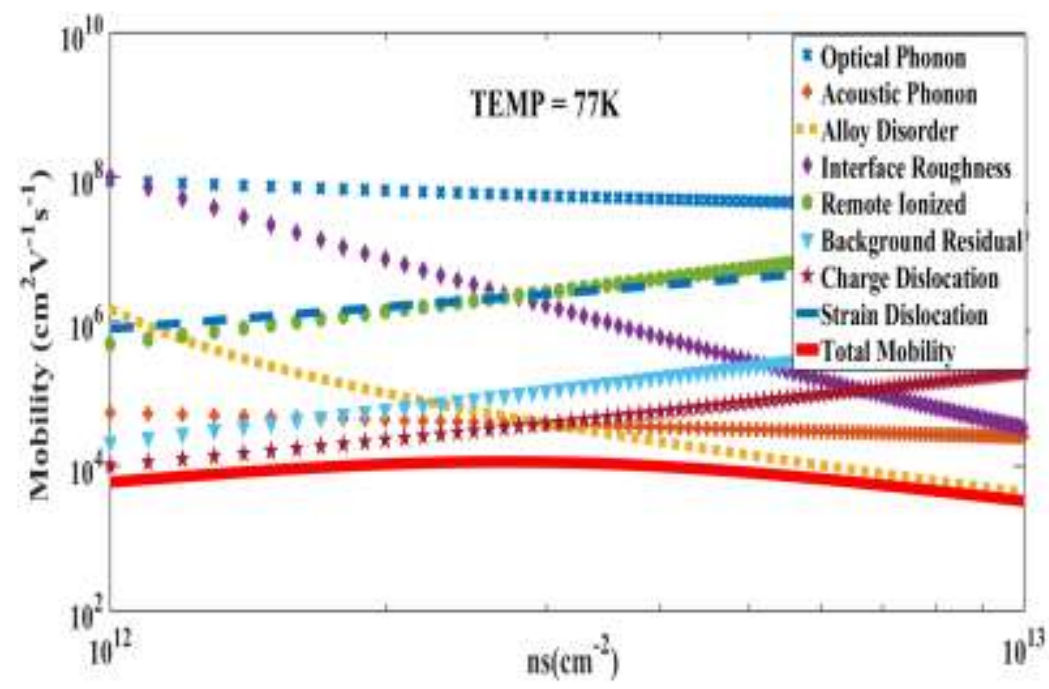

Fig. 11: Different mobilities plotted together against 2DEG density at 77K.

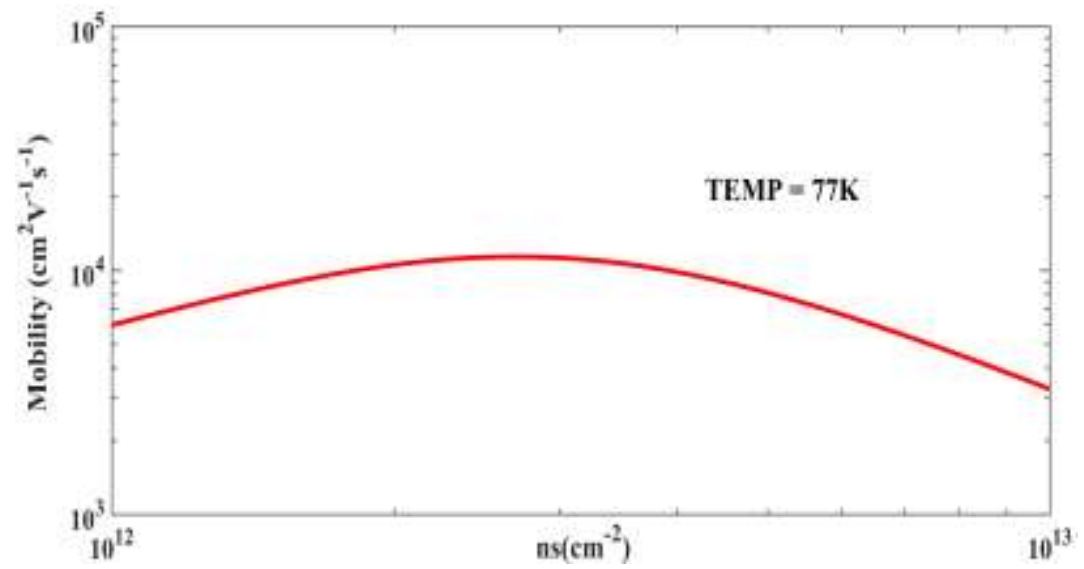

Fig. 12: Total mobility vs. 2DEG density at $77 \mathrm{~K}$.

\section{Conclusion}

In this work, various scattering mechanisms in $\mathrm{AlGaN} / \mathrm{GaN}$ HEMT were investigated and mathematical models for different scattering limited mobilities at room and low temperatures were developed. We calculated the total mobility as a function of 2DEG density. It is evident that temperature plays an important role in the mobility of AlGaN/GaN HEMT structure. Special interest in $\mathrm{AlGaN} / \mathrm{GaN}$ structures is the fact that there is a large improvement in the low-temperature mobility if a thin layer of AlN is sandwiched between the AlGaN/GaN layers (or the barrier is entirely AlN) [18]. The insertion of AlN causes the removal of alloy scattering, and thus shows that alloy scattering is a major scattering mechanism at low temperatures. There is also the scope of analysis of the carrier scattering phenomenon in the strain-free novel $\mathrm{Al}_{1-\mathrm{x}} \mathrm{In}_{\mathrm{x}} \mathrm{N} / \mathrm{GaN}$ structure and its comparison with the $\mathrm{AlGaN} / \mathrm{GaN}$ HEMT.

\section{Acknowledgements}

All research work presented in this paper is solely done in the Department of EEE, BUET. Authors of this work would like to thank Department of EEE, BUET for various supports during the preparation of this manuscript. 


\section{References}

[1] U. K. Mishra, P. Parikh and Y. F. Wu, "AlGaN/GaN HEMTs-an overview of device operation and applications," in Proceedings of the IEEE, vol. 90, pp. 1022-1031, 2002.

[2] M. J. Manfra, N. G. Weimann, J. W. P. Hsu, L. N. Pfeiffer, K. W. West, S. Syed, H. L. Stormer, W. Pan, D. V. Lang, S. N. G. Chu, G. Kowach, A. M. Sergent, J. Caissie, K. M. Molvar, L. J. Mahoney and R. J. Molnar, "High mobility $\mathrm{AlGaN} / \mathrm{GaN}$ heterostructures grown by plasma-assisted molecular beam epitaxy on semi-insulating GaN templates prepared by hydride vapor phase epitaxy," J. Appl. Phys., vol. 92, pp. 338, 2002.

[3] L. Pfeiffer, K. W. West, H. L. Stormer and K. W. Baldwin, "Electron mobilities exceeding $10^{7} \mathrm{~cm}^{2} / \mathrm{V} \mathrm{s}$ in modulationdoped GaAs," Appl. Phys. Lett., vol. 55, pp. 1888, 1989.

[4] W. Knap, S. Contreras, H. Alause, C. Skierbiszewski, J. Camassel, M. Dyakonov and J. L. Robert, "Cyclotron resonance and quantum Hall effect studies of the two-dimensional electron gas confined at the GaN/AlGaN interface," Appl. Phys. Lett., vol. 70, pp. 2123, 1997.

[5] R. Ishii, A. Kaneta, M. Funato, Y. Kawakami and A. A. Yamaguchi, "All deformation potentials in GaN determined by reflectance spectroscopy under uniaxial stress: Definite breakdown of the quasicubic approximation," Phys. Rev. $B$, vol. 81, pp. 155202, 2010.

[6] A. H. Davoody, E. B. Ramayya, L. N. Maurer and I. Knezevic, "Ultrathin GaN nanowires: Electronic, thermal, and thermoelectric properties," Phys. Rev. B, vol. 89, pp. 115313, 2014.

[7] K. Seeger, Semiconductor Physics, an Introduction, Berlin: Springer Verlag, 6th ed., 1999.

[8] B. L. Gelmont, M. Shur and M. Stroscio, "Polar optical-phonon scattering in three- and two-dimensional electron gases," J. Appl. Phys., vol. 77, pp. 657, 1995.

[9] J. P. Ibbetson, P. T. Fini, K. D. Ness, S. P. DenBaars, J. S. Speck and U. K. Mishra, "Polarization effects, surface states, and the source of electrons in AlGaN/GaN heterostructure field effect transistors," Appl. Phys. Lett., vol. 77, pp. 250, 2000.

[10] C. Wood and D. Jena, Polarization Effects in Semiconductors, US: Spinger Verlag, 2008.

[11] P. J. Hansen, Y. E. Strausser, A. N. Erickson, E. J. Tarsa, P. Kozodoy, E. G. Brazel, J. P. Ibbetson, U. Mishra, V. Narayanamurti, S. P. DenBaars and J. S. Speck, "Scanning capacitance microscopy imaging of threading dislocations in GaN films grown on (0001) sapphire by metalorganic chemical vapor deposition", Appl. Phys. Lett., vol. 72, pp. 2247, 1998.

[12] J. W. P. Hsu, M. J. Manfra, D. V. Lang, S. Richter, S. N. G. Chu, A. M. Sergent, R. N. Kleiman, L. N. Pfeiffer and R. J. Molnar, "Inhomogeneous spatial distribution of reverse bias leakage in GaN Schottky diodes," Appl. Phys. Lett., vol. 78, pp. 1685, 2001.

[13] A. F. Wright and J. Furthmüller, "Theoretical investigation of edge dislocations in AlN," Appl. Phys. Lett., vol. 72, pp. 3467, 1998.

[14] A. F. Wright and U. Grossner, "The effect of doping and growth stoichiometry on the core structure of a threading edge dislocation in GaN," Appl. Phys. Lett., vol. 73, pp. 2751-2753, 1998.

[15] J. S. Koehler, "A Calculation of the Changes in the Conductivity of Metals Produced by Cold-Work," Phys. Rev., vol. 75, pp. 106, 1949.

[16] C. Shi, P. M. Asbeck and E. T. Yu, "Piezoelectric polarization associated with dislocations in wurtzite GaN", Appl. Phys. Lett., vol. 74, pp. 573, 1999.

[17] K. Leung, A. F. Wright and E. B. Stechel, "Charge accumulation at a threading edge dislocation in gallium nitride," Appl. Phys. Lett., vol. 74, pp. 2495, 1999.

[18] I. P. Smorchkova, L. Chen, T. Mates, L. Shen, S. Heikman, B. Moran, S. Keller, S. P. DenBaars, J. S. Speck and U. K. Mishra, "AlN/GaN and (Al,Ga)N/AlN/GaN two-dimensional electron gas structures grown by plasma-assisted molecular-beam epitaxy," J. Appl. Phys., vol. 90, pp. 5196, 2001. 